Pensamiento Crítico Vol. 18 N$^{\circ}$ 2, pp. 7-18

\title{
Acerca de los estudios sobre China en el Perú
}

Carlos Aquino Rodríguez

\section{RESUMEN}

En este artículo se verá el estado de los estudios sobre China en el Perú. El país asiático cada vez es más importante para la economía peruana y el estudio sobre ese país, que tradicionalmente estaba concentrado en la inmigración china al Perú, en los ultimos años está viendo estudios sobre la relación comercial Perú-China también, además se ve un mayor interés en el idioma y cultura china. Todo esto indicaría que en el futuro habrá un mayor énfasis en la relación económica entre ambos países y se tendrá expertos en el tema con mayor conocimiento del idioma chino también.

Palabras claves: Estudios sobre China, inmigración china al Perú, relaciones Perú-China.

\section{ABSTRACT}

In this article a look is given to the state of china studies in Peru. The Asian country is becoming more important to the Peruvian economy now, and the traditional emphasis on studies of Chinese immigration to Peru is given way to studies about Peru-China economic relationship. In the future more studies on this theme will be seen with the addition that a group of experts with knowledge of Chinese language will appear.

Keywords: Studies on China, Chinese immigration to Peru, China-Peru relationship. 


\section{Pensamiento Crítico Vol. 18. No 2}

Como uno de los países con la más larga historia de inmigración china y con la más grande comunidad china en Latinoamérica, los estudios sobre China en Perú se enfocaron principalmente en la historia y el papel de los inmigrantes chinos en el Perú. Pero a medida que la presencia económica china crece y se convierte en el año 2011 en el mayor mercado de exportación de Perú, ahora la atención se centra en la relación económica y la influencia que China tiene en la economía del país. Por otra parte, con la creación en el Perú de varios Institutos Confucio en algunas Universidades, se demuestra que hay un gran interés en aprender el idioma chino y la cultura china, para comprender mejor al gigante económico por parte de las personas que quieren aprender las herramientas para hacer negocios con ese país.

En el presente artículo se presenta primero una breve introducción de la relación entre Perú y China, luego, una historia de los estudios chinos en el Perú, en la tercera parte se da una presentación de las investigaciones hechas sobre China, en la cuarta parte se presentan algunas iniciativas recientes de estudios sobre China y en las conclusiones se tratará de ver en qué direcciones estos estudios se desarrollarán.

\section{INTRODUCCIÓN}

Las relaciones entre Perú y China son de las más antiguas en Latinoamérica. El primer contacto entre estos dos países empezó en 1849, cuando inmigrantes chinos empezaron a venir al Perú. Desde ese año hasta 1872 cerca de 100,000 de ellos vinieron al país, mayormente de la parte sur de China, de la actual provincia de Guangdong. En ese tiempo Perú necesitaba fuerza laboral para las plantaciones de caña de azúcar y algodón a lo largo de la Costa (y luego en la extracción de guano y en la construcción del ferrocarril central del Perú). Había escasez de mano de obra porque los obreros que trabajan en las plantaciones, en su mayoría personas negras traídas antes como esclavos por los españoles en los siglos XVI al XVIII, se les dio libertad en 1845 (abolición de la esclavitud) y muchos de ellos dejaron los campos para irse a la ciudad.

Perú se convirtió en el primer país de Latinoamérica en recibir inmigración china a gran escala. China fue abierta a la fuerza por Inglaterra en 1840 luego de la guerra del opio y algunos de sus habitantes comenzaron a salir del país.

Pero la inmigración china al Perú termino repentinamente por un incidente en uno de los buques que los transportaba a Perú. En 1872 el barco peruano "María Luz", 


\section{Carlos Aquino Rodríguez}

durante una parada en el puerto de Yokohama, Japón, sufrió la deserción de un chino a bordo. Este se escapó lanzándose al mar, donde fue rescatado por la tripulación de un buque de guerra británico en el puerto. Se quejó ante el capitán del barco de los malos tratos en la nave peruana. De hecho, en el largo viaje desde China a Perú, las condiciones del viaje eran tan duras que a veces más de un tercio de la carga humana moría en el camino. ${ }^{1}$

Después de que el capitán del buque de guerra británico se quejara de esta situación ante las autoridades japonesas, se ordeno a la nave peruana regresar a los trabajadores a China. Luego de esto, el Gobierno Imperial chino de Qing prohibió la inmigración china al Perú.

Debido a este incidente, China y Perú iniciaron negociaciones para tener contactos oficiales, y en 1874 se establecieron relaciones diplomáticas.

Después de un paréntesis, el 02 de noviembre de 1971, se establecieron de nuevo las relaciones diplomáticas entre China y Perú. De esta manera Perú se convirtió en uno de los primeros países de América Latina en establecer relaciones diplomáticas con la ahora República Popular China.

Desde 1990 en adelante de nuevo más chinos emigran a Perú. Perú ahora es el hogar de la comunidad china más grande de América Latina.

Durante la década de 1990, las relaciones económicas empezaron a incrementarse. En 1993 el monto de exportaciones peruanas a China, fue solamente de 140 millones de dólares, pero en el 2003 estas alcanzaron los 676 millones de dólares. En el mismo periodo las importaciones de China aumentaron de 90 millones de dólares a 646 millones de dólares.

Perú se convirtió en 1998 en miembro del Foro de Cooperación Económica AsiaPacífico, APEC, uniéndose a China y otras economías asiáticas en esta importante organización y haciendo posible un mayor acercamiento entre los dos países. En 2009 se firmó un acuerdo de libre comercio entre ellos y este entró en vigor el año siguiente.

1 Fernando de Trazegnies: "En el país de las colinas de arenas", publicado por la Pontificia Universidad Católica del Perú, 1995. Hay una traducción china de este libro del año 1999 en Beijing. 


\section{Pensamiento Crítico Vol. 18. No 2}

En 2011 China se convirtió en el mayor socio comercial de Perú. Las exportaciones a China alcanzaron los 6.961 millones de dólares y las importaciones 6.321 millones de dólares en ese año.

En el año 2012 el comercio con China siguió aumentando (aunque las exportaciones peruanas al mundo no aumentaron) y las exportaciones a ese país ascendieron a 7.692 millones de dólares, con importaciones por valor de 7.795 millones de dólares. Ese año el $17 \%$ del total de las exportaciones de Perú fueron a China.

Las relaciones políticas entre Perú y China son muy fuertes y se han realizado varias visitas al más alto nivel entre ellos. En abril de 1991 Alberto Fujimori se convirtió en el primer presidente peruano en visitar China y fue un total de 4 veces durante su gobierno de diez años. En octubre de $1995 \mathrm{Li}$ Peng, el primer ministro chino en ese momento, hizo una visita oficial al Perú, convirtiéndose en el primer jefe de Estado chino en visitar nuestro país.

En junio de 2005 el presidente Alejandro Toledo, realizó una visita de Estado a China.

En el año 2008, cuando Perú fue sede de la Reunión de Líderes del foro APEC, el Presidente chino Hu Jintao visitó nuestro país.

El presidente del Perú Ollanta Humala viajó a China en abril de 2013 para una visita de Estado, donde fue recibido por el presidente Xi Jinping, convirtiéndose en uno de los primeros dignatarios extranjeros en reunirse con el recién elegido líder chino.

Además de estas visitas, los líderes de Perú y China se han reunido en varias ocasiones durante las reuniones de líderes del APEC en otros países.

\section{LA HISTORIA DE ESTUDIOS SOBRE CHINA EN EL PERÚ}

El tema más estudiado sobre China en el Perú es sobre la inmigración de los chinos a nuestro país. Como se sabe el Perú fue uno de los primeros países de América Latina en recibir inmigrantes de China en gran escala, y ahora tiene la comunidad china más grande de la región. 


\section{Carlos Aquino Rodríguez}

Se dice que quizás entre el $5 \%$ y el $10 \%$ de la población de Perú es de origen chino, pero muchos de ellos no tienen apellidos chinos y no tienen características físicas chinas. Lo que pasa es que ha habido una gran cantidad de matrimonios mixtos de chinos con peruanos y así los nombres y las características físicas se han perdido. Es interesante notar que los trabajadores chinos que llegaron al Perú durante el siglo XIX eran casi todos hombres. Como resultado los inmigrantes chinos tuvieron que casarse con mujeres peruanas.

También cabe señalar que debido a las duras condiciones en los campos en los que los trabajadores chinos tenían que trabajar al principio, algunos de ellos huyeron y fueron a lugares lejanos para evitar ser perseguidos. Algunos de ellos cambiaron sus apellidos para evitar ser capturados por las autoridades. Y, además, como algunos de ellos se establecieron en ciudades pequeñas, cuando se registraron ante las autoridades locales, y sobre todo cuando tenían sus hijos, en el momento de inscribir sus apellidos, las autoridades locales a menudo lo escribían mal en los documentos, certificados de nacimiento y de matrimonio, convirtiéndose sus nombres y apellidos en diferentes de los originales. ${ }^{2}$

Uno de los primeros y más prolíficos autores en el asunto de la inmigración china a Perú es Humberto Rodríguez Pastor. De hecho, él es un pionero en el estudio del tema. Sus libros incluyen "Hijos del Celeste Imperio en el Perú (1850-1900). Migración, Agricultura, Mentalidad y Explotación", publicado en 1989 por el Instituto de Apoyo Agrario. También ha publicado "Herederos del dragón" en el 2000 por el Fondo Editorial del Congreso del Perú.

Fernando de Trazegnies Granda publicó en 1994 "En el País de las colinas de arena: Reflexiones Sobre la Inmigración china en el Perú del S. XIX desde la Perspectiva del derecho", por la Pontificia Universidad Católica del Perú. Hay una traducción al chino de este libro. ${ }^{3}$ Este es uno de los libros más interesantes en la historia de la

2 He visto también por mí mismo estos errores en los nombres y apellidos en el caso de los japoneses que se asentaron en el Perú. Soy Traductor oficial de la lengua japonesa en el Perú y una de mis tareas es "corregir" la falta de ortografía de esos nombres en las partidas de nacimiento y otros documentos.

3 Fernando de Trazegnies Granda fue Ministro de Relaciones Exteriores de Perú y durante una visita a China en marzo del año 2000, mientras era Ministro, presentó su libro, traducido al chino en la Universidad de Beijing. Véase "DE TRAZEGNIES SE REUNIO CON VICEPRESIDENTE DE REP. POPULAR CHINA". http://cdn.preterhuman.net/texts/computing/gopher-archive/gopher.rcp.net.pe/noticias/andina/2000/ marzo/31 


\section{Pensamiento Crítico Vol. 18. No 2}

inmigración china al Perú y cuenta las vicisitudes que tuvieron que enfrentar para venir y vivir en el Perú.

También Isabelle Lausent-Herrera ha escrito varios libros y continúa investigando el tema de la inmigración china al Perú. Se publicó en 1992 su libro "La cristianización de los chinos en el Perú: Integración, Sumisión y Resistencia” por el Institut Français d'Etudes Andines. Ha publicado recientemente un interesante artículo sobre los chinos que llegan a Perú en las últimas dos décadas y su influencia en el barrio chino de Lima, capital del Perú. ${ }^{4}$

La influencia de los inmigrantes chinos en el Perú es muy conocida en varios temas y, por ejemplo una fuerte está en la comida peruana. Aquí los restaurantes chinos se llaman CHIFA. Esta palabra se dice que es una degeneración de la palabra china "Chifan" (吃饭). En Perú comemos mucho "Chaofan" “(炒饭), aquí se le llama" chaufa "o" arroz chaufa". Pero en Perú el Chaofan es diferente del arroz frito o Chaofan comido en China porque los restaurantes chinos o CHIFA ponen todo en él cuando preparan "arroz chaufa", no solo huevo frito, sino varios tipos de verduras, pollo, e incluso otros tipos de carne. Los CHIFA son numerosos en Perú y populares porque la comida es barata, deliciosa, abundante y estos la mayoría de veces están abiertos a los negocios a toda hora. Chaofan es también uno de los platos más populares que se consumen en el Perú.

Hay libros que describen la influencia de la comida china en la cocina peruana, como el uso de la salsa de soja, en Perú llamado "Siyau" (豉 油), o el uso del wok (镬), la sartén de fondo redondo versátil originaria de la región de Cantón de China. En el Perú algunas palabras en chino se pronuncian de la manera cantonesa porque la mayoría de los inmigrantes chinos que llegaron al Perú en el siglo 19 vinieron de esa región, o Guangdong.

Otra consecuencia de la presencia china en el Perú es que el arroz es el principal alimento en el país. Los peruanos comen arroz al menos dos veces por día, en el almuerzo y la cena. Es el país de América del Sur donde se consume más arroz que otros. Siendo Perú el país que dio al mundo la papa y el maíz este es un fenómeno muy interesante.

4 "The Chinatown in Peru and the Changing Peruvian Chinese community(ies)"en el Journal of Overseas Chinese, Volume 7, Issue 1, 2011, paginas 69 a 113,

http://halshs.archives-ouvertes.fr/docs/00/59/81/55/PDF/JCO_007_01_05-Lausent-Herrera.pdf 


\section{Carlos Aquino Rodríguez}

Un libro bien conocido sobre la comida china en el Perú o CHIFA es el de Mariella Balbi, una periodista, titulado "Los chifas en el Perú-Historia y recetas-", publicado en 1999 por la Universidad de San Martín de Porres. ${ }^{5}$

\section{INVESTIGACIONES SOBRE CHINA E INSTITUCIONES / CENTROS DE INVESTIGACIÓN DE ESTUDIOS SOBRE CHINA}

El "Centro de Estudios Orientales" de la Pontificia Universidad Católica del Perú6 es el centro más antiguo en el tema de los estudios sobre China y Asia en general, donde se dan algunos cursos, y se ha publicado sobre Asia y China. Creado en 1987 inició sus actividades el año siguiente. Se dictaban clases de idioma chino hasta el año 2009, cuando un Instituto Confucio inició sus actividades en la misma universidad y ahora las lecciones de chino se dan allí. En la actualidad el Centro de Estudios Orientales enseña los siguientes cursos sobre China: Introducción a la literatura china, Historia de la cultura China, e Introducción a las religiones chinas. ${ }^{7}$

Uno de los profesores que enseña allí es Guillermo Dañino Ribatto, que da el Seminario de Introducción a la literatura china. Es uno de los autores más prolíficos sobre la cultura y literatura china en el Perú. Escribió, por ejemplo, el libro "Esculpiendo Dragones: Antología de la Literatura de China", publicado por el Centro de Estudios Orientales en 1996, donde da a conocer al público peruano muchos poemas chinos que tradujo al español. Otro de sus libros es "Manantial de Vino. Poemas de Li Tai Po", publicado en 1998 también por el Centro de Estudios Orientales, un libro donde presentó y tradujo los poemas del gran poeta chino Li Bai ( $\mathrm{Li} \mathrm{Po}$ ). Guillermo Dañino pasó muchos años en China, y probablemente es el estudioso peruano que conoce mejor la cultura china y tiene el mayor conocimiento de la lengua china. ${ }^{8}$

Hay Institutos Confucio creados en los últimos años con el apoyo del gobierno de China. En la actualidad hay cuatro, dos en Lima, la capital, uno en el norte y otro

5 Véase el web site de la universidad, publicaciones del año 1999, "Los Chifas en el Perú" http://www.fcctp.usmp.edu.pe/facultad/libros-y-otras-publicaciones

6 http://ceo.pucp.edu.pe/ Yo solía enseñar aquí un "Seminario sobre economías del este asiático" desde 1994 al 2009 pero desde el año 2011 enseño esta materia en la Universidad Nacional Mayor de San Marcos en la Facultad de Ciencias Económicas

7 Véase las listas de materias que se enseñan en el Centro de Estudios Orientales http://ceo.pucp.edu.pe/ seminarios.htm

8 Véase su perfil en Wikipedia http://es.wikipedia.org/wiki/Guillermo_Da\%C3\%B1ino_Ribatto 


\section{Pensamiento Crítico Vol. 18. No 2}

en el sur del Perú. En Lima uno se encuentra en la Pontificia Universidad Católica del Perú ${ }^{9}$ y el otro está en la Universidad Ricardo Palma. ${ }^{10}$ En el norte del Perú en la ciudad de Piura ${ }^{11}$ existe un Instituto Confucio en la Universidad de Piura, creado en el año 2007. En el sur, en la ciudad de Arequipa, hay otro Instituto Confucio en la Universidad Católica de Santa María. ${ }^{12}$

Estos institutos enseñan principalmente la lengua china y también organizan eventos para promover la cultura china.

Este es un fenómeno muy interesante porque significa que en el Perú hay cuatro Institutos Confucio, un número grande de estos Institutos en América Latina. Esto demuestra un creciente interés en China y en el idioma chino en el Perú.

En el año 2012 la Universidad Alas Peruanas y la Fundación de Amistad Peruano China firmaron un acuerdo para crear un Instituto de Estudios Chinos, ${ }^{13}$ pero hasta ahora no ha habido alguna noticia adicional sobre esta iniciativa.

Recientemente, a finales del año 2012, se ha creado un Centro de Estudios Perú China en la Universidad del Pacífico en Lima.

\section{TEMAS DE ESTUDIOS SOBRE CHINA POR ACADÉMICOS Y PROFE- SORES PERUANOS Y UNA INTRODUCCIÓN ACERCA DE MI PROPIA INVESTIGACIÓN}

En los últimos años, como la presencia económica de China en el Perú y en la región de América Latina aumenta, el enfoque de los estudios sobre China es sobre todo en el aspecto económico.

9 El Instituto Confucio de aquí tiene un acuerdo de cooperación con la Universidad de Estudios Internacionales de Shanghái. http://confucio.pucp.edu.pe/

10 http://www.urp.edu.pe/iconfucio/presentacion.html

11 El Instituto Confucio de aquí tiene un acuerdo de cooperación con la Universidad Normal de Beijing. http://www.confucio.udep.edu.pe/

12 El Instituto Confucio de aquí tiene un acuerdo de cooperación con la Universidad de Estudios Extranjeros de Guangdong. http://www.ucsm.edu.pe/

13 De acuerdo con la página de Facebook de la Fundación de Amistad Peruano China. https://www.facebook. $\mathrm{com} / \mathrm{media} / \mathrm{set} /$ set $=$ a.371813849540460.97709.321181571270355\&type $=1$ 


\section{Carlos Aquino Rodríguez}

Por ejemplo, en el año 2009 dos autores, Cynthia Sanborn y Víctor Torres publicaron un libro con el título "La economía china y las Industrias extractivas: desafíos para el Perú", por la Universidad del Pacifico y Cooperaccion. ${ }^{14}$ El libro trata principalmente de las inversiones chinas en el sector minero del Perú.

En noviembre del año 2011 dos autores, Rubén Tang y Luis García-Corrochano presentaron un libro titulado "Política Exterior Peruana: Las Relaciones Entre el Perú y China» ${ }^{15}$, publicado por el Instituto de Estudios Internacionales y el Instituto Confucio de la Pontificia Universidad Católica del Perú. El libro ofrece una revisión de las relaciones entre Perú y China, incluso desde antes del establecimiento de relaciones diplomáticas en 1874.

En la segunda mitad del año 2012, la Universidad del Pacífico publicó un número especial de su revista "Apuntes", dedicada a las relaciones entre China y América Latina. La revista Apuntes $\mathrm{N}^{\circ} 71$ tiene como título: "Relaciones Comerciales y Políticas entre China, y Latinoamérica" ${ }^{16}$. Los artículos son de autores de otros países de la región de América.

En octubre del año 2012 un libro compilado por Richard Chuhue, Jing Li Na, y Antonio Coello, con el título de "La Inmigración China al Perú: Arqueología, Historia y Sociedad" 17 salió a la luz. Se trata de un libro interesante publicado por el Instituto Confucio de la Universidad Ricardo Palma y tiene 540 páginas, ya que junta los trabajos presentados en una Conferencia sobre los chinos en el Perú, que tuvo lugar un año antes. El libro abarca las presentaciones de casi 30 expertos en una amplia gama de campos, desde la investigación de la vida de un inmigrante chino en el siglo 19, al teatro chino en Lima, hasta el estudio del consumo de Chaofan en Perú (una lista de los temas tratados en el libro se puede ver en el enlace con el índice del libro en la nota 17).

14 http:/www.cooperaccion.org.pe/item/1-la-economia-china-y-las-industrias-extractivas-desafios-para-elperu.html

15 Ellibrofue presentadoen ocasión del 40 aniversario del establecimiento de relaciones diplomáticas modernas entre el Perú y China. https://www.facebook.com/notes/instituto-confucio-pucp/libro-pol\%C3\%ADticaexterior-peruana-las-relaciones-entre-el-per\%C3\%BA-y-la-china-de-venta/277119585664297

16 De los artículos en la revista sólo uno de ellos se refiere a Perú, sobre el Tratado de Libre Comercio suscrito entre Perú y China.

https://www.up.edu.pe/revista_apuntes/SitePages/tabla_contenido.aspx?idsec=Revista\% 20Apuntes\% 2071

17 http://summahistoriae.blogspot.com/2012/11/presentacion-del-libro-la-inmigracion.html 


\section{Pensamiento Crítico Vol. 18. No 2}

Ahora con respecto a mi propia investigación, desde que regresé al Perú en 1993 después de hacer estudios de posgrado en Japón me he dedicado a enseñar y escribir sobre el desarrollo económico de China y de la región de Asia Oriental. He publicado numerosos artículos sobre estos temas e incluso libros. En el año 2000 escribí un libro titulado "Introducción a la Economía Asiática", publicado por la Universidad Nacional Mayor de San Marcos. ${ }^{18}$ En este libro, varios capítulos están dedicados al análisis del desarrollo de la economía china.

Con respecto a mis artículos, por ejemplo he escrito para la revista "Pensamiento Crítico" No 14 del año 2010 del Instituto de Investigaciones Económicas de la Facultad de Economía de la Universidad Mayor de San Marcos uno sobre las relaciones entre China y Japón y en el "Pensamiento Crítico" No. 15 del año 2011 escribí otro artículo sobre China en la economía mundial y lo que significa para el Perú. ${ }^{19}$ El último artículo sobre China en esta revista lo publique en el Vol. 18 No. 1 de junio del 2013 bajo el titulo "El Rol de China en la economía asiática e implicancias para la encomia mundial" 20 También escribo en algunos diarios y revistas del Perú y del extranjero, por ejemplo he contribuido con algunos artículos al diario chino China Daily. ${ }^{21}$

También doy conferencias sobre economía china como la que di en septiembre del año 2012 en el "Colegio de Economistas de Lima". ${ }^{22}$ Además a menudo doy entrevistas a varios medios de comunicación de Perú y del extranjero sobre Asia y la economía china. ${ }^{23}$

También debo mencionar que participe invitado en marzo del 2013 en el Quinto Foro Mundial de los Estudios sobre China en la ciudad de Shanghái, organizada por la Academia

18 Todo el libro se puede leer en este enlace: http://economia.unmsm.edu.pe/Servicios/Publicaciones/Libros/ Libro2.htm

19 Artículo en la revista Pensamiento Crítico № 15, páginas 7 a 22. "La Economía china en la Coyuntura Económica Mundial y las implicancias para el Perú”, http://economia.unmsm.edu.pe/Organizacion/IIEc/ Archivos/RevistasIIE/PC_15/PC15_Completa.pdf

20 Este fue el resultado de una investigación que hice por 6 meses en la Universidad Tamkang en Taiwán, de febrero a agosto del 2012, gracias a una beca del Ministerio de Relaciones Exteriores de Taiwán.

21 Véase, por ejemplo, mi artículo: "La influencia de China crece en el 'patio trasero' de EE.UU"., China Daily, 25 de junio 2012

http://usa.chinadaily.com.cn/opinion/2012-06/25/content_15520252.htm

22 Conferencia "China y Asia en la actual situación económica" http://cel.org.pe/forocel/China\%20y\%20 Asia\%20en\%20al\%20actual\%20coyuntura\%20economica\%20fORO\%20CEL\%206-09.pdf

23 Por ejemplo ver la entrevista en Canal 7 del 27 de diciembre de 2013: http://www.tvperu.gob.pe/noticias/ economia/negocios/53638-especialistas-destacan-sociedad-comercial-con-china.html 


\section{Carlos Aquino Rodríguez}

de Ciencias Sociales de Shanghái y la Oficina de Información de la Municipal de Shanghái. En este evento, presenté un trabajo sobre las relaciones entre Perú y China. ${ }^{24}$

En la Facultad de Ciencias Económicas de la Universidad Nacional Mayor de San Marcos donde enseño, dicto un "Seminario sobre las economías asiáticas", donde hablo sobre el desarrollo económico de China y otros países asiáticos. Por lo que sé, es la única universidad en el Perú donde un tema como éste es enseñado, y esa materia ya se ha incorporado como parte del Syllabus del Departamento de Economía.

En otro aspecto, es interesante notar que el gobierno peruano ha comenzado a dar becas para estudiar en China. CONCYTEC, la institución oficial para la ciencia y la tecnología está ofreciendo financiamiento para los estudios de Maestría, Doctorado, formación especial, y del idioma chino en universidades de ese país. ${ }^{25}$ Uno de mis alumnos consiguió una de estas becas y se fue a China en septiembre del 2013 para hacer un curso de doctorado en una Universidad en Beijing sobre economía china. Gracias a esto en el futuro tendremos académicos peruanos que han estudiado en China y entiendan el idioma chino.

También hay que hacer notar que durante la visita del presidente Ollanta Humala en abril del 2103 a China se abrió el Centro de Estudios Peruanos en la Universidad Normal de Hebei ${ }^{26}$, el primero de este tipo en China. Debe tenerse en cuenta que esta es la Universidad que estableció un acuerdo de cooperación con la Universidad Ricardo Palma de Perú para establecer un Instituto Confucio en esta. Así que será interesante ver en el futuro el intercambio de académicos entre Perú y China que estudien la relación entre ambos países.

Por último cabe mencionar que algunas universidades en Perú están estableciendo acuerdos de cooperación con universidades de China, como se mencionó en el caso de la creación de los Institutos Confucio en el Perú, pero no sólo en este tema. Por ejemplo,

24 Ver el web site del Fórum: http://www.chinastudies.org.cn/english.htm

25 Véase: "CONCURSO NACIONAL DE BECAS DE ESTUDIOS DE DOCTORADO, MAESTRIA, PERFECCIONAMIENTO DE ESPECIALIDADES O APRENDIZAJE DEL IDIOMA CHINO EN UNIVERSIDADES DE LA REPÚBLICA POPULAR CHINA 2013 - 2014", http://portal.concytec.gob.pe/ index.php/ FONDECYT-becas-china-2013.html

26 "Se establece primer centro de estudios peruanos en universidad china", ver en la página web de la Embajada de China en Lima, Perú, http://www.embajadachina.org.pe/esp/xwss/t1029233.htm 


\section{Pensamiento Crítico Vol. 18. No 2}

la Universidad Nacional Mayor de San Marcos tenía un acuerdo de cooperación con la Universidad de Estudios Internacionales de Beijing. ${ }^{27}$

\section{CONCLUSIONES}

Como se ha visto, en los últimos años el enfoque de los estudios sobre China en el Perú va en la dirección de una mejor comprensión de la relación económica de ambos países. Los estudios sobre la inmigración china con seguridad continuarán dada la larga historia de esa relación y el impacto que tienen en la vida peruana, desde la comida a otros campos.

Pero otro cambio que está ocurriendo es el fuerte interés por la lengua y la cultura china. Hasta ahora había muy pocas personas que entendían bien el idioma chino, un elemento vital para hacer negocios con China o comprender mejor y hacer mejor investigación sobre China. Con la puesta en marcha de varios Institutos Confucio más personas están aprendiendo el idioma chino, y no sólo en estos Institutos Confucio el lenguaje está siendo enseñado, sino también en otros lugares. ${ }^{28}$ Así que en el futuro, es seguro que van a salir nuevos investigadores con conocimientos de la lengua china.

Asimismo, como se mencionó antes el gobierno peruano, en coordinación con el gobierno chino, está ofreciendo más becas a estudiantes e investigadores para ir a estudiar a China. Además cabe mencionar que poco a poco los estudiantes chinos parecen estar llegando a Perú para estudiar el idioma español, y también están interesados en la oportunidad de hacer negocios con el Perú. Una ventaja para ellos en venir aquí es que los chinos son bienvenidos por los peruanos precisamente por la presencia de una gran comunidad china.

Sin duda, todos estos esfuerzos de los investigadores ayudarán a comprender mejor las relaciones económicas y también será una razón para aumentar esta aun más. Una nueva generación de investigadores con conocimientos de la lengua china y con experiencia de haber estado en China también seguro ampliara el tema de las investigaciones.

27 Para obtener una lista de los acuerdos de cooperación que la Universidad Nacional Mayor de San Marcos tiene, véase este enlace: http://www.unmsm.edu.pe/coop/contenidob1e4.html?pag1=6

28 Sólo por mencionar uno, por ejemplo, en la Universidad Nacional Mayor de San Marcos, en su Centro de Idiomas, el idioma chino se viene enseñando desde el año 2012. http://ceidunmsm.com/idiomas/index. php?option $=$ com_content\&view $=$ article\&id $=349 \&$ Itemid $=145$ 